\title{
The Assessment of Aortic Pulse Wave Velocity Using 4D Flow Magnetic Resonance Imaging: Methods Comparison
}

\author{
Sophia Houriez--Gombaud-Saintonge ${ }^{1,2}$, Elie Mousseaux ${ }^{3}$, Ioannis Bargiotas ${ }^{1}$, Alain De \\ Cesare $^{1}$, Thomas Dietenbeck ${ }^{1}$, Kévin Bouaou ${ }^{1}$, Alban Redheuil ${ }^{1}$, Gilles Soulat ${ }^{3}$, Umit Gencer ${ }^{3}$, \\ Damian Craiem $^{4}$, Yasmina Chenoune ${ }^{2}$, Nadjia Kachenoura ${ }^{1}$ \\ ${ }^{1}$ Sorbonne Universités, UPMC Univ Paris 06, INSERM, CNRS, Laboratoire d'Imagerie \\ Biomédicale, Paris, France \\ ${ }^{2}$ ESME SUDRIA, Esme Research Lab, PARIS, France \\ ${ }^{3}$ Hopital Européen Georges Pompidou, PARIS, France \\ ${ }^{4}$ Universidad Favaloro-CONICET, IMeTTyB, Buenos Aires, Argentina
}

\begin{abstract}
Purpose: Compare various methods of transit time (TT) and consequently aortic Pulse Wave Velocity (aoPWV) estimation from 4D MRI (aoPWV=aortic length/TT), in terms of associations with age and Bramwell-Hill (BH aoPWV). Method: We studied 43 healthy subjects $(48 \pm 17 y$ rs.) who had aortic 4 DFlow MRI. Three strategies were used to estimate aoPWV: (S1) using flow curves in two aortic locations to calculate TT (ascending (AA) and distal descending aorta (dDA)) as a 2D-like strategy, (S2 and S3) using flow curves of the entire aortic path-line between dDA and AA to estimate TT with various methods: cross-correlation; Fourier and Wavelet or aoPVW by fitting a plan on the systolic upslope of flow curves. Results: Expected associations with age were found for the three strategies with strongest correlations for $3 D$-like strategies than 2D-like strategy ( $S 2: r=0.75$, S3: $r=0.72, p<0.001, \quad S 1: \quad r=0.55, p<0.001)$. Similar results were found for associations with $\mathrm{BH}$ aoPWV. Best results were obtained using the TT waveletbased approach. Conclusion: Low temporal resolution of 4 DFlow is compensated by aortic $3 D$ coverage, leading to strong associations of aoPWV with age and $\mathrm{BH}$ aoPWV. Key words: aorta; stiffness; pulse wave velocity; flow sensitive 4D MRI.
\end{abstract}

\section{Introduction}

Aortic stiffness is an early sign of remodelling and functional change in arterial hemodynamic and a marker of cardiovascular aging [1]. Its association with coronary heart disease, atherosclerosis and elevated mortality demonstrates its clinical usefulness [2]. Aortic stiffness is usually assessed using carotid-femoral pulse wave velocity (PWV), which is the speed of pressure pulse propagation within the arterial tree located between the carotid and femoral arteries. PWV is defined by the distance travelled by the pressure wave between two anatomical locations, divided by the transit time (TT) spent by the wave to travel between the same locations. Cardiovascular magnetic resonance imaging (MRI) offers an excellent anatomical coverage and allows with its anatomical and velocity sequences an accurate estimation of aortic geometry (length, diameters, volumes) as well as blood flow derived indices. In particular, the two dimensional phase contrast MRI with velocity encoding in a single through plane z-direction (2D-MRI) has been used in several studies for the estimation of aortic PWV (aoPWV) using arch length and flow waves derived from the acquisition plane crossing perpendicularly both the ascending (AA) and the descending (DA) aorta [1]. Flow waves have been used to calculate TT as the time spent by blood flow to travel between AA and DA, while considering time-based (crosscorrelation) [1] and frequency-based (Fourier, wavelets) approaches [3, 4]. More recently, an MRI sequence with velocity encoding in the three directions and with a full three-dimensional anatomical coverage (4D flow MRI) during the cardiac cycle has been developed, opening a new area of research in terms of cardiovascular complex flow visualization and quantification. Indeed, 4D flow MRI has some advantages over 2D flow MRI such as: 1) the more accurate estimation of the measured velocities, since all three spatial velocity directions were taken into account rather than the single velocity perpendicular to the acquisition plan, 2) The straightforward positioning of the acquisition volume throughout the aorta and the heart prevent from a possible variability of positioning a plan in a precise anatomical location in patients with variable aortic geometry. These 4D flow advantages are 
obtained at the expense of lower spatial and temporal resolutions as well as a longer acquisition time. The aim of this study is to evaluate three different strategies of PWV estimation from 4D flow MRI while assuming that the full 3D coverage would counterbalance the lower temporal resolution.

\section{Material and methods}

\subsection{Study population and data acquisition}

Population comprised 43 healthy subjects ranged from 20 to 79 years (mean age $48 \pm 17$ years), without history of cardiovascular disease. Protocol has been approved by the local ethics committee and all participants have signed an informed consent.

All subjects had 2D cine and a 4D flow MRI exams on a 3.0 Tesla magnet (GE Healthcare) with electrocardiographic and respiratory prospective gating. For the 2D-MRI cine acquisition, an axial plane was positioned perpendicular to AA and DA at the level of the bifurcation of the pulmonary artery and the SSFP data were acquired during breath-hold using the following average scan parameters: repetition time $(\mathrm{TR})=3.4 \mathrm{~ms}$, echo time $(\mathrm{TE})=1.4$ ms, flip angle $=50^{\circ}$, views per segment $=26$, slice thickness $=6 \mathrm{~mm}$, pixel spacing $=0.76 \times 0.76 \mathrm{~mm}^{2}$, matrix $=224 \times 192$ and temporal resolution $=10 \mathrm{~ms}$ after applying a view sharing technique. For 4D flow the three-directional velocity encoding was equal to $250 \mathrm{~cm} / \mathrm{sec}$ and scan parameters were as follows: echo time $=1.7 \mathrm{~ms}$, repetition time $=4.3-4.4 \mathrm{~ms}$, flip angle $=15^{\circ}$, voxel size $=1 \times 1.48 \times 2.38 \mathrm{~mm}^{3}$ frames per cardiac cycle $=50$. To enhance the signal to noise ratio, a single dose of contrast agent has been used for 4Dflow.

For 4Dflow, phase offset correction and velocity dealiasing have been applied. Besides, to improve the quality of aortic segmentation, a phase contrast angiogram (3D PCA) was computed while combining the modulus and velocities averaged over time. Segmented PCA was used to delineate velocities and to extract aortic centerline and thus aortic length. Plans perpendicular to centerline (Fig. 1) were used to calculate flow curves.

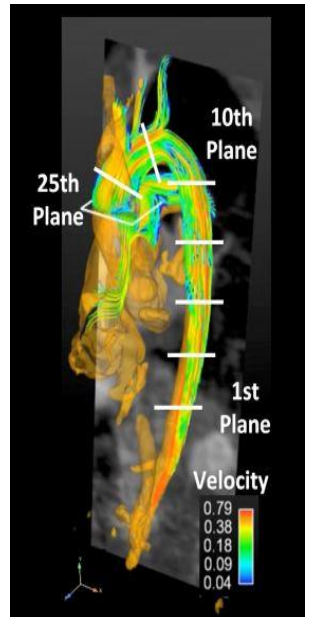

Figure 1. Aortic 4D flow image with plan positioning.
Central blood pressures have been measured within the magnet and carotid systolic (SBP) and diastolic (DBP) pressures as well as pulse pressure (PP) were recorded. AA and DA lumen area were automatically delineated for all phases of the cardiac cycle on SSFP images using the validated ArtFun software (U.1146 Inserm/UPMC). Then, local AA pulse wave velocity was calculated from AA distensibility according to the Bramwell-Hill model $(\mathrm{BH}$ aoPWV $=1 / \sqrt{\text { blood density } \times \text { distensibility }})$, where distensibility is calculated as (AA systolic area-AA diastolic area)/(AA diastolic area $\cdot \mathrm{PP})$. The same formula is then applied for DA and BH aoPWV is calculated as the average of AA and DA values.

\subsection{Pulse Wave velocity Estimation}

MRI aoPWV is the ratio of the distance between two arterial locations $(\Delta \mathrm{D})$ and the transit time (TT) (usually for 2D MRI, aoPWV is measured between $\mathrm{AA}$ and DA) i.e. aoPWV $=\Delta \mathrm{D} / \mathrm{TT}$.

Three strategies of PWV computation are used on 4D flow data: Strategy 1 is a $2 \mathrm{D}$ strategy and the aoPWV is computed as the ratio of aortic length and TT between AA and distal DA (dDA). Strategy 2 (Fig. 2.a). uses the full 3D anatomical coverage and has been previously presented in $[5,6]$. It calculates TT and aortic length along the centerline between the dDA plan and all the planes equally positioned between $\mathrm{dDA}$ and AA, resulting a distance/time dot plot. Then, the aoPWV is calculated as the inverse of the slope of the linear fit of such dot plot. Strategy 3 (Fig. 2.b): has been previously presented in [7]. It also uses the full coverage of the aorta but no TT is needed. Indeed aoPWV is calculated as the slope of the plan fitting the systolic upslope of the flow curves extracted from the plans positioned perpendicular to centreline, distributed equally between $\mathrm{dDA}$ and AA.

For strategy 1 and 2, TT is calculated as the temporal gap between two flow curves using 3 different approaches: 1) the first is a time-domain approach based of Cross Correlation (TTc). TTc is equal to the time shift between two flow curves which maximizes their correlation. 2 and 3 ) the two remaining methods Fourier (TTf) and Wavelet (TTw) are frequency-based and were previously shown to be more robust to a low temporal resolution [3, 4] .For both methods the group delay method was used to estimate the harmonic phase difference between two flow curves. TTf and TTw are energy-weighted sum of harmonics phases. Of note, while the entire curve is needed for TTf, only the systolic upslope is used for the TTc and TTw to avoid the deleterious effect of wave reflections. 

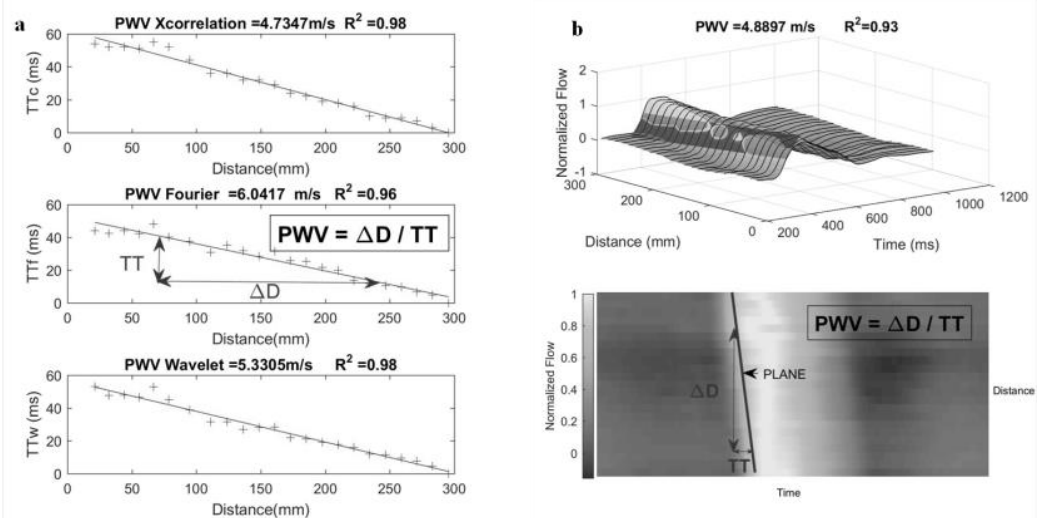

Figure 2. Strategy 2(a) and 3(b) to compute the pulse wave velocity PWV. TT: transit time, and $\Delta \mathrm{D}$ : distance

\subsection{Statistical analysis}

Bland-Altman analysis was performed for comparisons between all the computed 4D flow aoPWV against $\mathrm{BH}$ aoPWV and mean bias and confidence interval (CI) (mean bias $\pm 1.96 \times$ standard deviation) are provided. Linear regression was used to study the relation between $\mathrm{BH}$ aoPWV and age. A multivariate model including age and possible confounders such as body mass index (BMI) systolic blood pressure (SBP), heart-rate (HR) was performed. Statistical analyses have been performed on STATA.

\section{Results}

Average values of aoPWV for subjects $<50$ years and $\geq 50$ years are summarized in Figure 3 for all methods indicating higher aoPWV in the elderly. While for subjects $<50$ years average aoPWV are nearly equivalent for all methods, such values are more variable for subjects $\geq 50$ years. Such differences can be explained by the fact that TT estimation is more challenged in the in elderly in whom aoPWV is higher as a consequence of low TT that gets closer to or lower than time resolution.

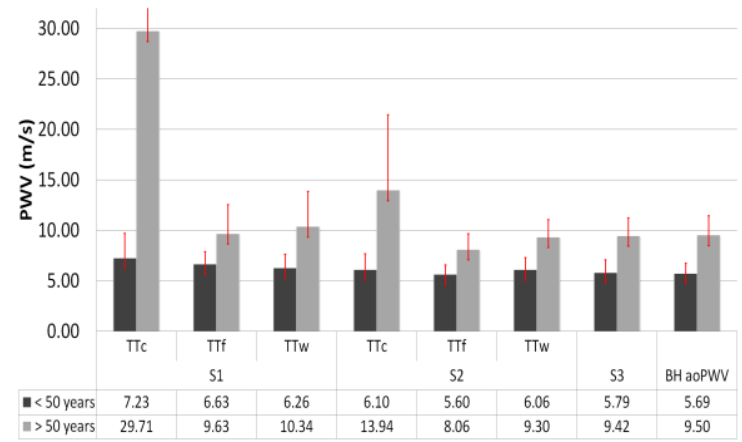

Figure 3: Average values of aoPWV measures for subjects $<50$ years and $\geq 50$ years.

\subsection{Comparison to theoretical PWV}

Bland and Altman comparison of our aoPWV values against the theoretical $\mathrm{BH}$ aoPWV resulted in low mean bias and reasonable intervals of agreement for all methods (S1-TTw: $0.699 \mathrm{~m} / \mathrm{s}$ [-0.349: 1.748]; S1-TTf: is $0.566 \mathrm{~m} / \mathrm{s}$ [-0.528:1.659]; S2-TTw: 0.107 $\mathrm{m} / \mathrm{s} \quad$ [-0.520: 0.735]; S2-TTf: $-0.718 \mathrm{~m} / \mathrm{s} \quad[-1.511$ : $0.075]$; S3: $0.019 \mathrm{~m} / \mathrm{s}$ [-0.770:0.807]), except for those based on TTc: (S1-TTc: $10.226 \mathrm{~m} / \mathrm{s}$ [1.051:19.400], S2-TTc: $2.286 \mathrm{~m} / \mathrm{s}$ [0.199:4.372]).

\subsection{Correlation with Age}

Correlation coefficients corresponding to the linear regression of all 4D flow aoPWV measures with age and $\mathrm{BH}$ aoPWV are summarized in Figure 4. The three PWV computed with the 2D like strategy (S1) show the weakest correlation with both age $(0.35<\mathrm{R}<0.55)$ and BH aoPWV $(0.26<\mathrm{R}<0.48)$. Highest correlations were found for the $3 \mathrm{D}$ like strategies S2 (age:0.53<R<0.75; BH aoPWV: $0.51<\mathrm{R}<0.70$ ); $\mathrm{S} 3$ (age: $\mathrm{R}=0.72 ; \mathrm{BW}$ aoPWV:R=0.58), with a superiority of S2 - wavelet (TTw) and S3.

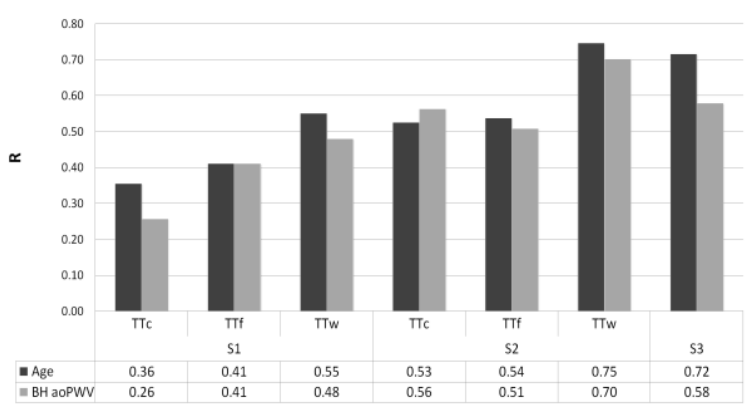

Figure 4. Coefficient of linear correlation $\mathrm{R}$ with Age and $\mathrm{BH}$ aoPWV for all the pulse wave velocity.

For the aoPWV methods with strongest associations with age, a multivariate analysis was 
performed and summarized in Table 2 indicating that their relationship with age is independent of SBP, HR and BMI. Of note, HR is also an independent correlate of aoPWV.

Table 1: A. Linear Regression Strategy2-Wavelet (S2 $2_{\mathrm{TTw}}$ ) and Strategy 3 (S3) aoPWV with age. B. multivariate model with adjustment for body mass index (BMI) systolic blood pressure (SBP), heartrate (HR). $\left({ }^{* * *} \mathrm{p}<0.001,{ }^{* *} \mathrm{p}<0.05,{ }^{*}: \mathrm{p}<0.01\right)$.

\begin{tabular}{c|c|ccccc}
\hline & A & \multicolumn{5}{|c}{ B } \\
\hline PWV & R $^{2}$ & Age & BMI & SBP & HR & R $^{2}$ \\
\cline { 2 - 7 } S2 & $\mathbf{0 . 5 6}^{* * * *}$ & $0.1^{* * * *}$ & 0.04 & 0.01 & $0.06^{*}$ & $\mathbf{0 . 6 4}$ \\
S3 & $\mathbf{0 . 5 2}^{* * * *}$ & $0.1^{* * *}$ & 0.04 & 0.07 & $0.02^{* *}$ & $\mathbf{0 . 6 2}$ \\
\hline
\end{tabular}

\section{Discussion}

The main findings of our work are that: 1) methods previously used for 2D aoPWV estimation do not translate directly to 4D Flow data, 2) methods of aoPWV estimation should take into account the full aortic coverage to result in more robust aoPWV estimates from 4D Flow data, 3) the previously demonstrated higher robustness to low temporal resolution of frequency-based methods for TT estimation, as compared to time-domain methods is confirmed in our data.

While the 2D based approaches remain relatively robust in subjects $<50$ years, they fail in some elderly subjects with stiff arteries and high aoPWV as revealed by the occurrence of inconsistent aoPWV values (>20 m/s). This might be explained by: 1) the inability of 2D based approaches to capture low TTs in data with such low temporal resolution, 2) the low quality of the 4D flow data segmentation through the cardiac cycle which affects the quality of flow rate curves crucial for a relevant TT estimation. Indeed, the high quality of the modulus image in $2 \mathrm{D}$ flow MRI and the availability of validated and reproducible dynamic segmentation techniques provide high quality flow rate curves than those usually derived from 4D flow data.

The aoPWV estimation is more challenging in elderly in whom PWV increases as a consequence of TT lowering, although, such the exploration of such patients is of high clinical importance. Accordingly, the optimisation of MRI temporal resolution is essential to collect reliable aoPWV measurements. With technological advances, MRI devices are able to acquire $2 \mathrm{D}$ velocity encoded data with reasonable temporal resolutions (up to $10 \mathrm{~ms}$ ). Regarding 4D Flow MRI, the high anatomical coverage and the realistic 3D velocity encoding are unfortunately counterbalanced by low temporal resolution in the actual versions of the sequences. To overcome such low resolution, TT estimation should consider velocity and thus flow data of the entire aorta in which continuous evaluations would smooth TT results by minimizing local effect of noise.

\section{Conclusion}

The aortic pulse wave velocity can be assessed from 4D flow MRI in young and elderly subjects, while taking advantage of the full anatomical coverage of the aorta. The obtained PWV results are consistent with physiological knowledge as revealed by the strong and independent associations with age. 4D flow MRI is an original sequence, which can in the near future help for a comprehensive evaluation of the aorta throughout the quantification of its geometry, function and blood flow hemodynamics. Validation studies along with robust processing technique are however needed to achieve such aim.

\section{Acknowledgements}

We would like to acknowledge the ECOSSUD project number A15S04 (France-Argentina) and the FRM project ING20150532487.

\section{References}

[1] Redheuil A, Yu W-C, Wu CO, et al. Reduced Ascending Aortic Strain and Distensibility. Hypertension 2010; 55: 319-326.

[2] Laurent S, Cockcroft J, Van Bortel L, et al. Expert consensus document on arterial stiffness: methodological issues and clinical applications. Eur Heart J 2006; 27 : 2588-2605.

[3] Taviani V, Hickson SS, Hardy CJ, et al. Age-related changes of regional pulse wave velocity in the descending aorta using Fourier velocity encoded M-mode. Magn Reson Med 2011; 65: 261-268.

[4] Bargiotas I, Mousseaux E, Yu W-C, et al. Estimation of aortic pulse wave transit time in cardiovascular magnetic resonance using complex wavelet cross-spectrum analysis. J Cardiovasc Magn Reson 2015; 17: 65.

[5] Spottiswoode B, Stalder AF, Gulsun MA, et al. Fast semiautomated analysis of pulse wave velocity in the thoracic aorta using high temporal resolution 4D flow MRI. $J$ Cardiovasc Magn Reson 2013; 15: P87.

[6] Markl M, Wallis W, Brendecke S, et al. Estimation of global aortic pulse wave velocity by flow-sensitive 4D MRI. Magn Reson Med 2010; 63: 1575-1582.

[7] Markl M, Wallis W, Strecker C, et al. Analysis of pulse wave velocity in the thoracic aorta by flow-sensitive fourdimensional MRI: Reproducibility and correlation with characteristics in patients with aortic atherosclerosis. $J$ Magn Reson Imaging 2012; 35: 1162-1168.

Sophia Houriez--Gombaud-Saintonge. 93 boulevard de l'hôpital, 75013, PARIS sophia.houriez--gombaud-saintonge @etu.upmc.fr 